\title{
Imprinted survival genes preclude loss of heterozygosity of chromosome 7 in cancer cells
}

\author{
Arnoud Boot,' Jan Oosting,' Noel FCC de Miranda,' Yinghui Zhang,' Willem E Corver,' Bob van de Water,'2 \\ Hans Morreau' and Tom van Wezel',* \\ I Department of Pathology, Leiden University Medical Center, Leiden, The Netherlands \\ 2 Division of Toxicology, Leiden Academic Center for Drug Research, Leiden University, The Netherlands
}

*Correspondence to: T van Wezel, Department of Pathology, Leiden University Medical Center, Leiden, The Netherlands. E-mail: t.van_wezel@/umc.nl

\begin{abstract}
The genomes of a wide range of cancers, including colon, breast, and thyroid cancers, frequently show copy number gains of chromosome 7 and rarely show loss of heterozygosity. The molecular basis for this phenomenon is unknown. Strikingly, oncocytic follicular thyroid carcinomas can display an extreme genomic profile, with homozygosity of all chromosomes except for chromosome 7. The observation that homozygosity of chromosome 7 is never observed suggests that retention of heterozygosity is essential for cells. We hypothesized that cell survival genes are genetically imprinted on either of two copies of chromosome 7, which thwarts loss of heterozygosity at this chromosome in cancer cells. By employing a DNA methylation screen and gene expression analysis, we identified six imprinted genes that force retention of heterozygosity on chromosome 7. Subsequent knockdown of gene expression showed that CALCR, COPG2, GRB10, KLF14, MEST, and PEG10 were essential for cancer cell survival, resulting in reduced cell proliferation, $G_{1}$-phase arrest, and increased apoptosis. We propose that imprinted cell survival genes provide a genetic basis for retention of chromosome 7 heterozygosity in cancer cells. The monoallelically expressed cell survival genes identified in this study, and the cellular pathways that they are involved in, offer new therapeutic targets for the treatment of tumours showing retention of heterozygosity on chromosome 7.

Copyright @ 2016 Pathological Society of Great Britain and Ireland. Published by John Wiley \& Sons, Ltd.
\end{abstract}

Keywords: imprinted cell survival genes; haploidization; oncocytic thyroid cancer; allelic-specific gene expression; CALCR; COPG2; GRB10; KLF14; MEST; PEG10

Received II March 2016; Revised 2I May 2016; Accepted 24 May 2016

No conflicts of interest were declared.

\section{Introduction}

Chromosomal instability is widespread in cancer. A distinction can be made between whole-chromosomal instability, leading to numerical changes of chromosomes, and structural chromosomal instability. Structural chromosomal instability is the result of successive breakage-fusion-bridge cycles and is characterized by the loss of large segments of chromosomes [1]. Whole-chromosomal instability can occur as a result of chromosomal mis-segregation during mitosis [2,3]. Replication stress is a common denominator of both forms of chromosomal instability [4]. Overall, chromosomal instability is favourable for tumour cells, as it aids in inactivating tumour suppressor genes by losing alleles, as well as amplifying chromosomal regions which contain tumour-promoting genes.

In contrast to most other tumour types, differentiated thyroid carcinomas generally present stable genomic profiles. The most frequent chromosomal aberration in papillary thyroid carcinomas affects chromosome 22 , where loss of one copy is observed in $9 \%$ of samples [5]. Exceptions to the stable genomic profiles are the oncocytic follicular thyroid carcinomas. These represent $3-4 \%$ of all thyroid malignancies and have been shown to be a particularly aggressive thyroid cancer subtype [6-8].

Single nucleotide polymorphism (SNP)-array profiling revealed widespread loss of heterozygosity in the genomes of oncocytic follicular thyroid carcinomas [9-12]. These tumours show either near-haploid genomes or near-homozygous genomes as a result of genomic endoreduplication. The near-homozygous genomes probably result from chromosomal mis-segregation events $[10,13,14]$. The minimal signature of the near-homozygous genomes in thyroid cancer was defined as homozygosity of chromosomes 1, 2, 3, 8, and 11 [10]. Notably, homozygosity on chromosome 7 was never observed and allelic balance at this chromosome is always retained [9-11]. This suggests that while homozygosity of most chromosomes is tolerated or favoured during thyroid cancer progression, 
the loss of one of the alleles of chromosome 7 is selected against.

Near-homozygous genomes are also observed in a fraction of other endocrine malignancies such as adrenal cortical carcinomas and parathyroid carcinomas [10]. Also, acute lymphoblastic leukaemias, chondrosarcomas, gliosarcomas, and glioblastomas occasionally present with near-homozygous genomes [13-16]. Interestingly, whole chromosomal loss is almost always observed, as opposed to the loss of parts of chromosomes due to chromosomal rearrangements.

We postulate that monoallelic expression of cell survival genes on the paternal and maternal copies of chromosome 7 explains the retention of heterozygosity at this chromosome. Thus, loss of either copy of chromosome 7 would result in a complete loss of gene expression. The term 'cell survival genes' refers to any gene that is essential for cellular survival [17,18]. Identification of cell survival genes is crucial for the discovery of novel therapeutic targets. In a background of somatic alterations, synthetic lethal interactions might be explored to minimize the impact on healthy cells [19].

In this study, we performed genome-wide DNA methylation analysis with the aim of identifying monoallelically methylated genes. Monoallelic gene expression analysis on the latter was followed by siRNA-mediated knockdown for functional characterization. We determined that CALCR, COPG2, GRB10, KLF14, MEST, and PEG10 are imprinted cell survival genes that obligate retention of heterozygosity at chromosome 7 .

\section{Materials and methods}

\section{Patient material}

Snap-frozen thyroid surgical specimens were collected from the biobank of the Pathology Department, Leiden University Medical Center (LUMC). The thyroid cancer sample set consisted of 19 previously described samples [10], expanded with four medullary thyroid carcinomas and three oncocytic follicular thyroid carcinomas. These patients were enrolled under an earlier study protocol [20], approved by the Institutional Review Board of the LUMC. This study has been registered at ClinicalTrials.gov (https://clinicaltrials.gov/; No NCT00887107). Informed written consent was obtained from all patients in the study. Fully anonymized snap-frozen normal thyroid tissues were collected according to the medical ethical guidelines described in the Code Proper Secondary Use of Human Tissue established by the Dutch Federation of Medical Sciences (https://www.federa.org/). Normal tissues with a high lymphocyte content were excluded from the study. Samples and analyses are listed in Table S1 of the supplementary material.

\section{Nucleic acid isolation}

Frozen tissues were sectioned and H\&E-stained to check the tumour cell percentage, histology, and tissue integrity for both the tumour and the normal thyroid epithelium. Patches of infiltrating lymphocytes were removed before sectioning for nucleic acid isolation.

DNA was isolated using the NucleoSpin ${ }^{\circledR}$ Tissue kit (Macherey-Nagel, Düren, Germany), and RNA using Nucleospin ${ }^{\circledR}$ RNA columns (Macherey-Nagel). RNA integrity was determined using the Agilent 2100 Bioanalyzer RNA 6000 Nano kit (Agilent Technologies Inc, Santa Clara, CA, USA). Samples with a RIN score below 5.0 were excluded, and the median RIN value was 8.2 .

\section{Infinium HumanMethylation450 BeadChips}

$500 \mathrm{ng}$ of DNA was bisulfite-converted using the EZ DNA Methylation-Gold ${ }^{\mathrm{TM}}$ kit (Zymo Research, Irvine, CA, USA). Quality control of bisulfite-converted DNA and array hybridization were performed at ServiceXS (GenomeScan BV, Leiden, The Netherlands).

DNA methylation array data processing and analysis were performed using R3.02. DNA methylation array data were processed using the LUMI package and normalized using the BMIQ function [21,22].

Vigorous filtering was applied to reduce the influence of background signals by removing all sites with a total intensity less than the median total intensity of all sites. 217164 sites remained for further analysis. QC showed that the distribution of probes over the chromosomes was unaffected by application of the $50 \%$ intensity filter (supplementary material, Figure S1). The DNA methylation data have been submitted to GEO under accession number GSE77804.

\section{Genotyping}

SNP genotyping was performed using KASPar assays (LGC Genomics, Hoddesdon, Herts, UK). All the primers used in this study are listed in Table $\mathrm{S} 2$ of the supplementary material.

\section{Gene expression analysis}

A series of 14 normal thyroid samples and 25 thyroid cancer samples were used for qPCR analyses. The thyroid cancer set included 12 of the 14 samples included in the DNA methylation analysis. The thyroid cancer samples were subdivided into medullary thyroid cancers $(N=4)$ and non-medullary thyroid cancers $(N=21)$.

cDNA synthesis and qPCR were performed as described previously [23]. Gene expression was normalized to the geometric mean of three reference genes, CPSF6, HNRNPM and TBP.

For allele-specific expression analysis, $2 \mu \mathrm{l}$ of $25 \times$ diluted cDNA was used for the KASPar assay. Using the $C q$ values obtained for both alleles, the allelic dosage was calculated similarly to the Pfaffl method for relative gene expression [24]. The allelic dosage observed in the cDNA was then compensated for the amplification difference between the two alleles in the DNA sample. 


\section{siRNA knockdown}

siRNAs were obtained from the Dharmacon siRNA library (GE Dharmacon, lafayette, CO, USA). siRNA knockdown was performed using a SMARTpool containing four siRNAs targeting the same gene. A list of the siRNAs used is included in the supplementary material, Table S3. siRNA targeting KIF11 was used as a positive control, and the negative controls used were siRNA targeting $G A P D H$, and two scrambled controls. For siRNA knockdown, cells were seeded in 96-well plates at 5000 cells per well. Transfection was performed $24 \mathrm{~h}$ after seeding using $50 \mathrm{nM}$ siRNA with $0.3 \%$ INTERFERin (Polyplus-Transfection SA, Illkirch-Graffenstaden, France). Medium was refreshed $24 \mathrm{~h}$ after transfection. The biomass present at $48 \mathrm{~h}$ post-transfection was measured using the sulphorodamin-B assay. Biomass was normalized to the biomass measured at the time of transfection [25].

\section{RNA isolation after siRNA knockdown}

RNA isolation after siRNA transfection was performed using TRIzol (Thermo Scientific, Rockford, IL, USA). TRIzol extracts of quadruplicates were pooled. The aqueous phase was isolated and further purified using Nucleospin ${ }^{\circledR}$ RNA columns (Macherey-Nagel).

\section{Apoptosis}

Apoptosis was detected $48 \mathrm{~h}$ after siRNA transfection using the Caspase 3 Assay kit (Sigma-Aldrich, St Louis, MO, USA; Cat No CASP3F). The apoptosis test was performed in triplicate.

\section{Cell cycle analysis}

Cell cycle analysis was performed $48 \mathrm{~h}$ after transfection. Cells from six wells of a 96-well plate were pooled and prepared for FACS analysis as described previously [26]. Cells were analysed using an LSR II flow cytometer (Becton Dickinson BV, Breda, The Netherlands). During data storage, all events were included. Data were analysed using WinList 7.1 (Verity Software House, Topsham, ME, USA). All FACS results were repeated in three independent experiments.

\section{Results}

\section{Genome-wide DNA methylation profiling}

In order to identify monoallelically expressed cancer cell survival genes on chromosome 7 , we set out to identify hemi-methylated loci assuming that this is the major mechanism that regulates monoallelic gene expression.

DNA methylation profiles were generated by using the Infinium HumanMethylation450 BeadChip on thyroid cancer samples that were previously reported with near-homozygous genomes $(N=7)$ or genomically stable profiles $(N=7)[11]$. The cut-offs for unmethylated, hemi-methylated, and methylated sites were determined based on the density distribution of the methylation levels ( $\beta$-values, Figure 1A). Sites with $\beta$-values between 0.3 and 0.7 were defined as hemi-methylated and consequently, all probes with a $\beta$-value below 0.3 were considered unmethylated and those above 0.7 methylated. Density plots for the individual samples are included in the supplementary material, Figure S2.

Additionally, we compared the differences in genomic distribution of hemi-methylated sites between the near-homozygous and genomically stable sample groups. The methylation status of defined regions required the concordance of at least five samples per group. As expected, hemi-methylation on homozygous regions of the near-homozygous sample group was less frequent compared with the genomically stable group.

\section{Identification of hemi-methylated sites on chromosome 7}

We selected all sites on chromosome 7 which were hemi-methylated in all 14 thyroid cancer samples. After removal of sites with false-positive hemi-methylation, the remaining 42 sites were plotted on the chromosome (Figure 1B). A detailed explanation of quality control steps performed in the DNA methylation analysis and filtering of false-positive hemi-methylation is included in the supplementary material, Supplementary materials and methods.

We identified three distinct clusters of hemi-methylated sites which are all known to be imprinting clusters: chr7p12.2, chr7q21.3, and chr7q32.2, harbouring a total of 15 genes that have been described previously to be imprinted [27,28]. The $\beta$-values of the 42 loci for the individual samples are plotted in Figure S3 of the supplementary material.

\section{Gene expression at imprinting clusters} on chromosome 7

The expression levels of the genes located in the imprinting clusters on chromosome 7 were measured in 14 normal thyroid tissues and 24 thyroid cancers. We found that CALCR, COPG2, COPG2IT1, KLF14, MEST, PEG10, and PPPIR9A were up-regulated in thyroid cancer compared with normal thyroid tissue (Figure 2). Interestingly, we found that $C A L C R$ and $K L F 14$ were exclusively expressed in thyroid cancer samples, but not in normal tissues.

Of the 15 genes identified as imprinted on chromosome 7 , we found that 12 genes were expressed in the thyroid cancer samples (supplementary material, Figure S4). For the remaining three genes, CPA4, $D D C$, and MESTIT1, expression could not be detected in the majority of thyroid cancer samples. MESTIT1 expression was detected at low levels in several normal thyroid samples. Its expression seems to originate from parafollicular cells as it was restricted to medullary thyroid cancer samples $(N=4)$, which are known to develop from that cell type.

Altogether, gene expression was confirmed in all three imprinting clusters on chromosome 7 in the majority 

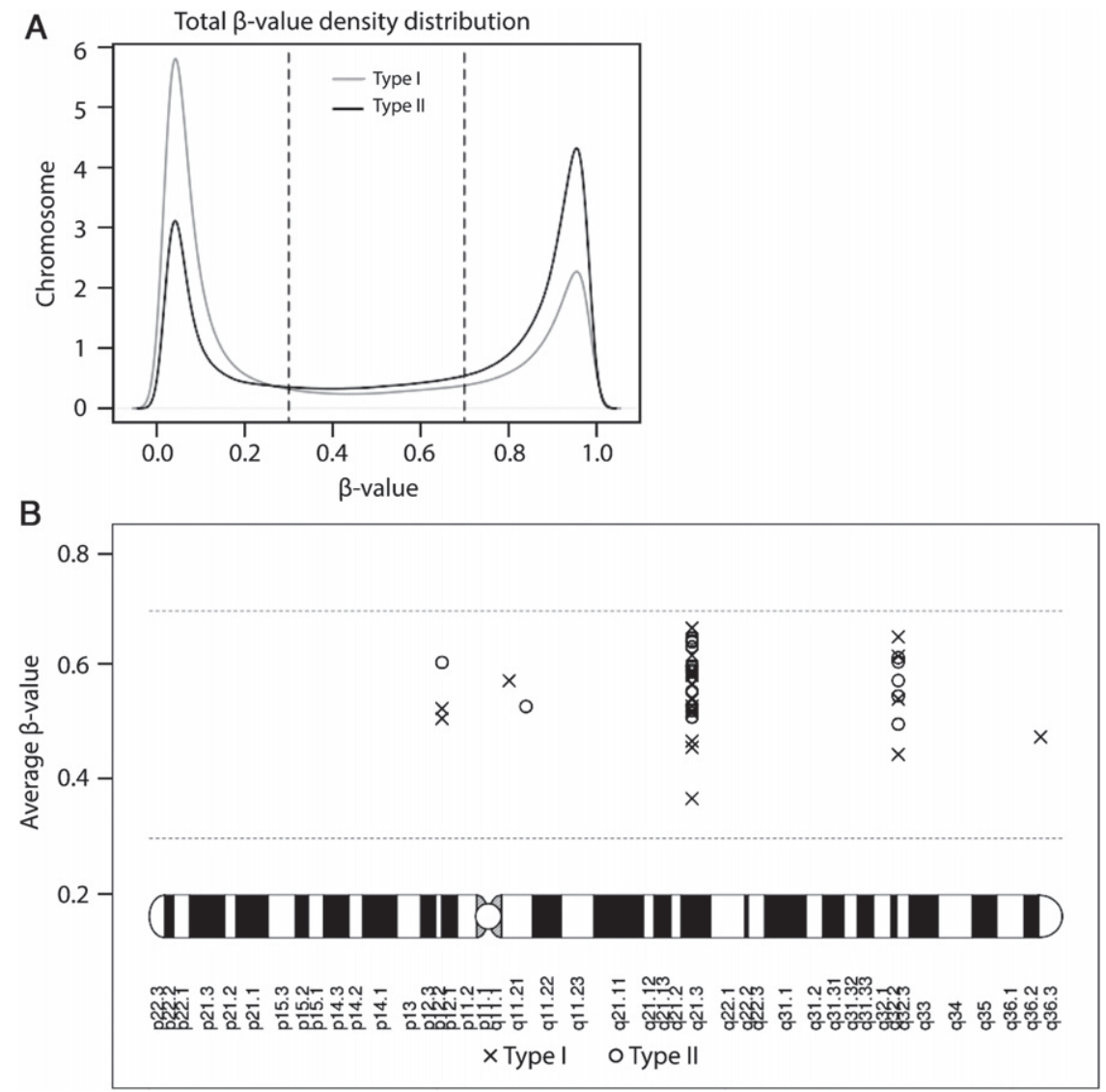

Figure 1. DNA methylation analysis. (A) Density distribution of $\beta$-values of all the samples used in this study. Cut-offs for hemi-methylation were set at $\beta$-values of 0.3 and 0.7 . Sites with $\beta$-values below 0.3 were considered unmethylated and above 0.7 methylated. (B) Distribution of hemi-methylation on chromosome 7 in all thyroid cancer samples. After removal of sites with false-positive hemi-methylation as detected in the adrenal cortical carcinomas, three clusters of hemi-methylation were identified.

of thyroid cancer samples, providing further support for the hypothesis that these genes are driving the retention of heterozygosity on chromosome 7 (summarized in Table 1). Furthermore, cancer-specific up-regulation of seven of these genes supports their relevance for cancer cells.

\section{Allele-specific expression analysis of imprinted genes}

To verify whether the genes on chromosome 7 are indeed regulated by imprinting in thyroid cancer cells, we performed allele-specific expression (ASE) analysis. These assays targeted coding SNPs (cSNPs) that were selected based on high minor allele frequency in the general population. ASE results were classified as monoallelic, predominantly monoallelic, or biallelic. The cut-off for predominantly monoallelic expression was set at an allelic dosage of 1:3 [29].

GRB10, located at chr7p12.2, was assayed targeting an exon shared by all isoforms. The analysis showed that both normal and thyroid cancer samples exhibited biallelic expression of GRB10 (Figure 3A). As GRB10 displays isoform-specific imprinting, we also assayed a cSNP located in the 5'UTR of two specific isoforms [30,31]. This revealed monoallelic expression of these isoforms (Figure 3A).
Of the genes located at the chr7q21.3 imprinting cluster, monoallelic expression of PEG10 and predominantly monoallelic expression of $C A L C R$ were detected. For the CALCR KASPar assay, an allele amplification bias was observed in DNA genotyping, while allelic balance at chromosome 7 is known for these samples (Figure 3B). After correction of this bias CALCR showed predominantly monoallelic expression (Figure 3C, D).

ASE of COPG2 and COPG2IT1 was determined by sequencing, as common cSNPs are not known in these genes. DNA sequencing of $551 \mathrm{bp}$ of the 3'UTR of COPG2 failed to reveal heterozygous SNPs in any of the normal or cancer samples. Two samples were found to be heterozygous for COPG2IT1. cDNA analysis showed that both samples have monoallelic gene expression of COPG2IT1 (Figure 3 F). KASPar analysis of KLF14 and MEST identified monoallelic expression of KLF14 and biallelic expression of MEST in all heterozygous samples. We investigated whether isoform-specific imprinting occurs in MEST, as observed for GRB10, since the cSNP targeted for ASE detection is shared by all isoforms (Figure 3E). Sequencing of rs146617166, located in the 5'UTR of two MEST isoforms, was performed, showing monoallelic expression in both heterozygous samples (Figure 3G). 

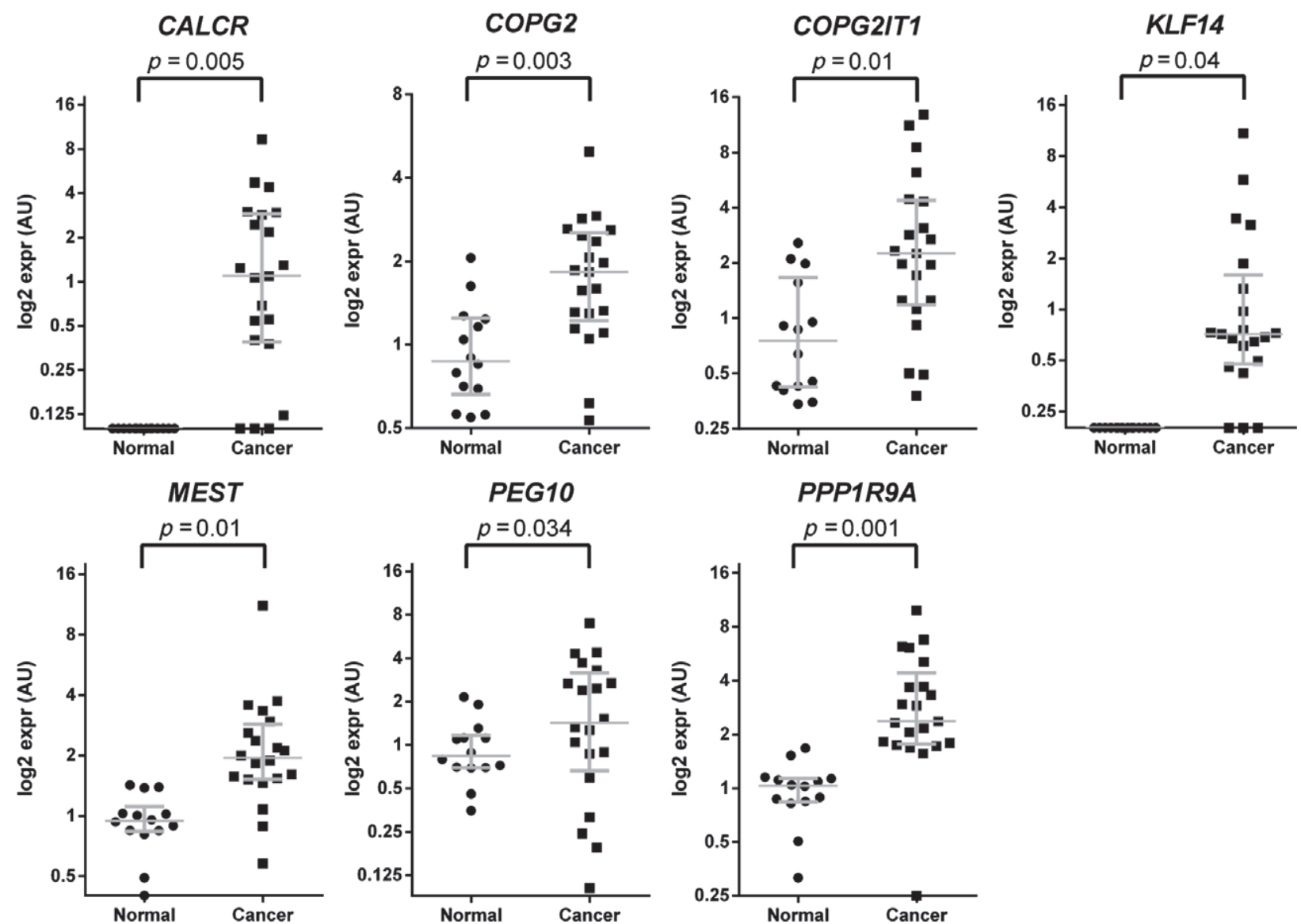

Figure 2. Gene expression analysis. Gene expression results for the seven genes located at the chromosome 7 imprinting clusters showing increased gene expression in the thyroid cancer samples compared with normal thyroid epithelium. Medullary thyroid cancer samples were not included in this analysis.

Table 1. Imprinted genes on chromosome 7 in thyroid cancer

$\begin{array}{lclll}\text { Gene } & \text { Imprinting cluster } & \text { Expressed allele* } & \text { Gene expression } & \text { ASE result } \\ \text { DDC } & 7 \mathrm{p} 12.2 & \text { Isoform-specific } & \text { Not expressed } & \text { - } \\ \text { GRB10 } & 7 \mathrm{p} 12.2 & \text { Isoform-specific } & \text { Down-regulated } & \text { Isoform-specific } \\ \text { CALCR } & 7 q 21.3 & \text { Maternal } & \text { Only in thyroid cancer } & \text { Predominantly monoallelic } \\ \text { DLX5 } & 7 q 21.3 & \text { Maternal } & \text { Stable } & \text { Biallelic } \\ \text { MAG/2 } & 7 q 21.3 & \text { Maternal } & \text { Stable } & \text { Biallelic } \\ \text { PEG10 } & 7 q 21.3 & \text { Paternal } & \text { Up-regulated } & \text { Monoallelic } \\ \text { PPP1R9A } & 7 q 21.3 & \text { Maternal } & \text { Up-regulated } & \text { Biallelic } \\ \text { SGCE } & 7 q 21.3 & \text { Paternal } & \text { Stable } & \text { Biallelic } \\ \text { TFPI2 } & 7 q 21.3 & \text { Maternal } & \text { Stable } & \text { Biallelic } \\ \text { COPG2 } & 7 q 32.2 & \text { Paternal } & \text { Up-regulated } & \text { Undetermined } \\ \text { COPG2IT1 } & 7 q 32.2 & \text { Paternal } & \text { Up-regulated } & \text { Monoallelic } \\ \text { CPA4 } & 7 q 32.2 & \text { Maternal } & \text { Not expressed } & - \\ \text { KLF14 } & 7 q 32.2 & \text { Maternal } & \text { Only in thyroid cancer } & \text { Monoallelic } \\ \text { MEST } & 7 q 32.2 & \text { Paternal } & \text { Up-regulated } & \text { Isoform-specific } \\ \text { MESTIT1 } & 7 q 32.2 & \text { Paternal } & \text { Not expressed } & -\end{array}$

*As reported in the literature [28].

Genes expressed in less than 50\% of the non-medullary thyroid cancer samples were excluded from ASE analysis.

\$No heterozygous cSNPs were available for ASE analysis of COPG2.

To further investigate the isoform-specific imprinting of the GRB10 and MEST genes, we employed a nested KASPar approach. A schematic representation of the isoform-specific amplicons is shown in Figure S5 of the supplementary material. Three different amplification patterns were identified by KASPar analysis for every assay: monoallelic, predominant monoallelic expression and biallelic expression. The endpoint fluorescence scatter plot and exemplary amplification curves are shown in Figure 4A and 4B, respectively. The ASE calls are depicted in Figure 4C for GRB10 and in Figure 4D for MEST.

Both GRB10 and MEST showed monoallelic or predominantly monoallelic expression in all samples for one and two of their isoforms, respectively. These corresponded to the same isoforms that were 
A

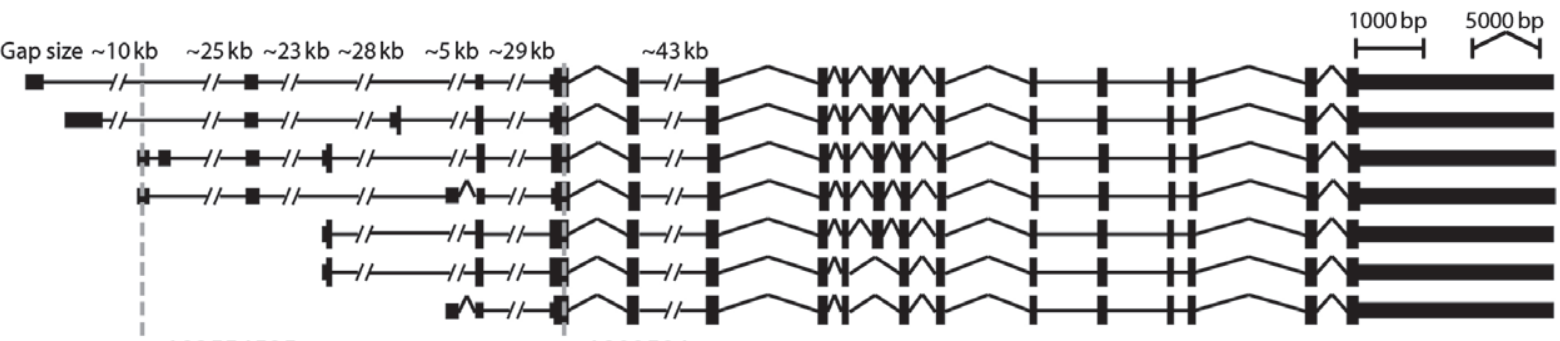

rs 182554585

B

rs2301680 KASPar Allelic bias tested on DNA

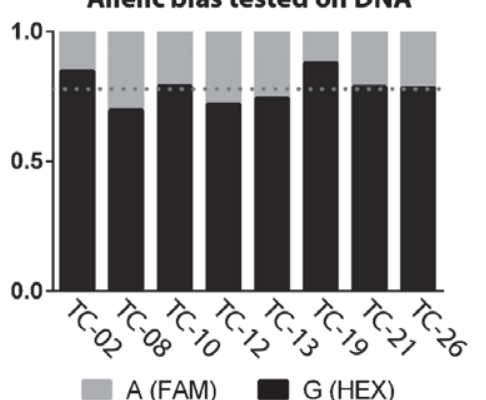

C

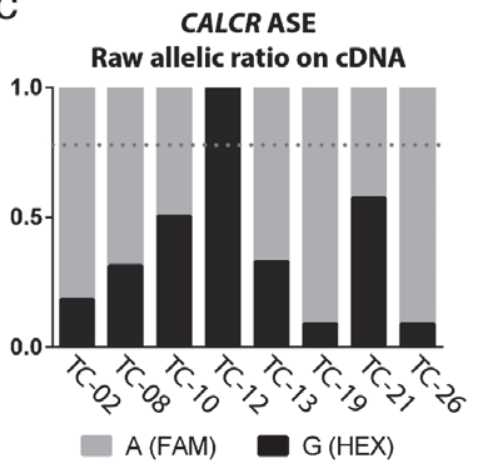

D

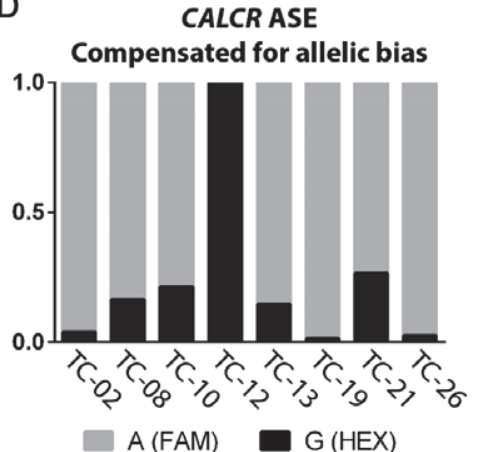

E

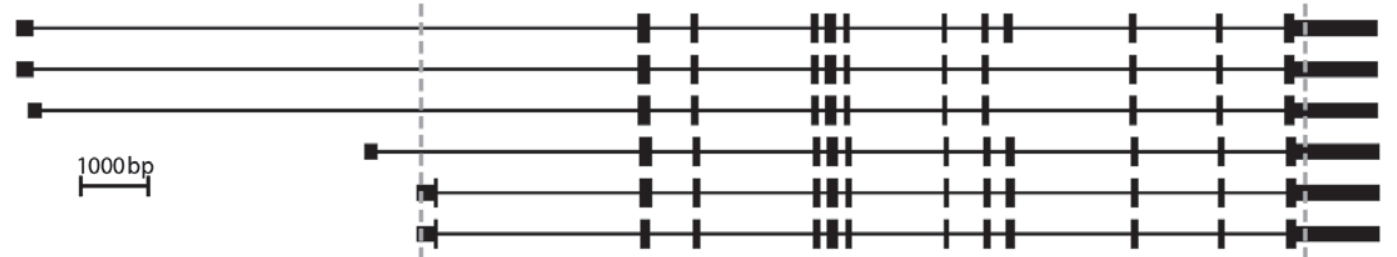

rs146617166

rs1050582

F

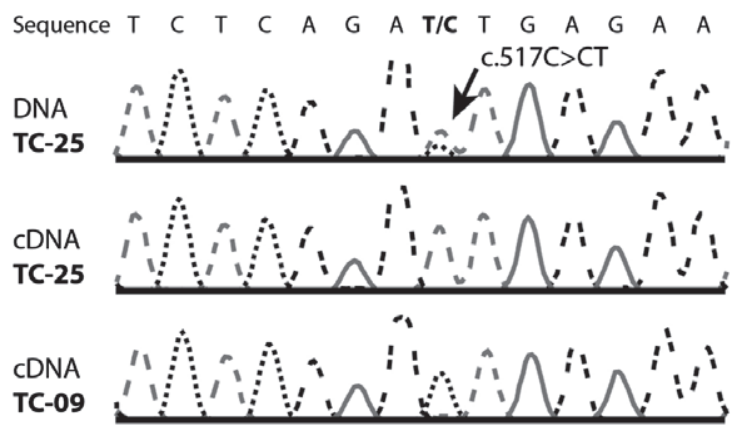

G

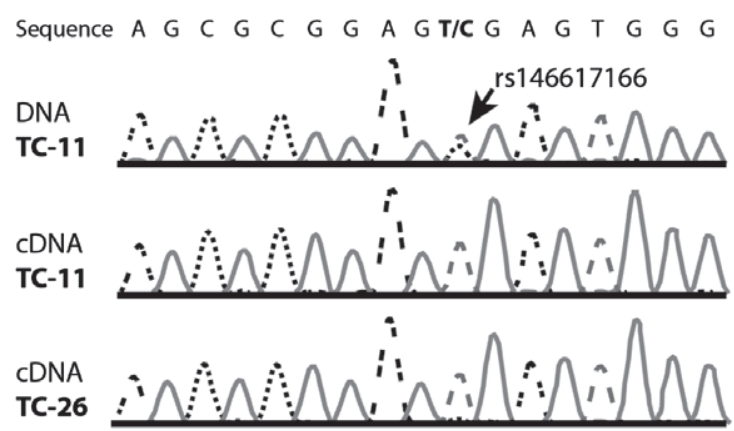

Figure 3. ASE results. (A) GRB10 isoforms with utilized CSNPs. The most pronounced differences are found on the $5^{\prime}$ end of GRB10. rs182554585 was used to test ASE of a subset of GRB10 isoforms, whereas rs1800504 could only be employed to test ASE of all isoforms together. (B-D) CALCR ASE analysis using KASPar genotyping. (B) Allelic ratio for rs2301680 in the DNA of heterozygous samples. The average is indicated by the dotted line. (C) Allelic dosage for rs2301680 in the cDNA before compensating for assay-specific allelic amplification bias. (D) Allelic dosage for rs 2301680 after compensation for assay-specific allelic bias. TC-21 was found to show unbalanced expression of $C A L C R$; the remaining seven heterozygous samples all showed monoallelic or predominantly monoallelic expression. (E) MEST isoforms. The MEST locus encodes six known isoforms. Analysis of rs 1050582 is transcribed in all isoforms, whereas rs 146617166 is specific for two of the isoforms. (F, G) ASE sequencing results for COPG2IT1 (F) and MEST-rs146617166 (G), located in the 5'UTR of two of the MEST isoforms. For each gene, the top panel shows the sequence trace for one of the heterozygous DNA samples. The bottom two panels show the cDNA sequence traces for both heterozygous samples.

analysed by the isoform-specific cSNP analysis described previously.

To summarize, we identified six monoallelically expressed genes on chromosome 7. CALCR, COPG2, COPG2IT1, GRB10, KLF14, MEST, and PEG10 are therefore potential cell survival genes involved in the retention of chromosome 7 heterozygosity. An overview of the gene expression and ASE conclusions is shown in Table 1. Detailed information on the cSNPs tested and the numbers of samples found monoallelically expressing each gene is included in Table S4 of the supplementary material.

\section{siRNA-mediated knockdown of imprinted genes}

To investigate whether the genes showing ASE play a role in tumour cell survival, we performed siRNA-mediated knockdown in TT2609-C02 cells, 
A Endpoint fluorescence nested KASPar

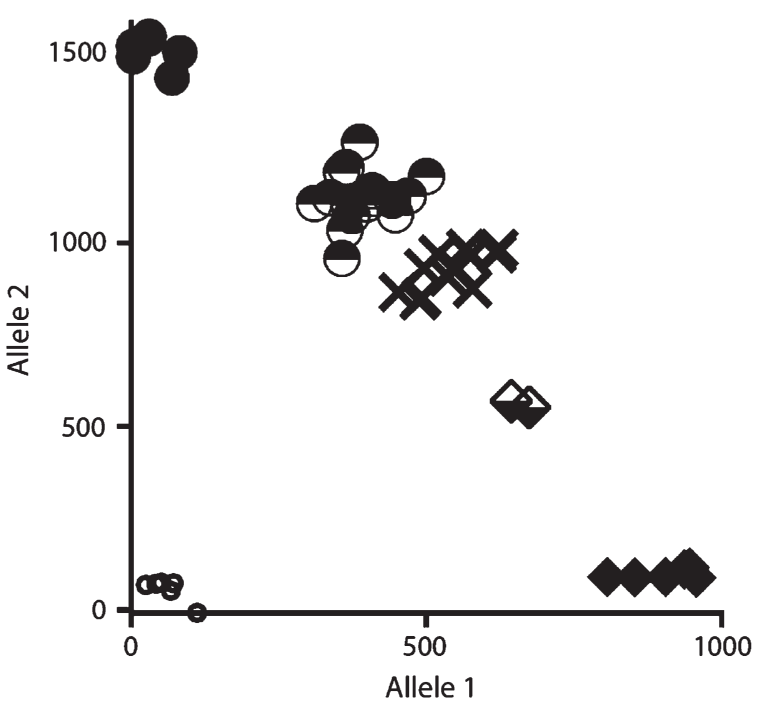

\section{B}

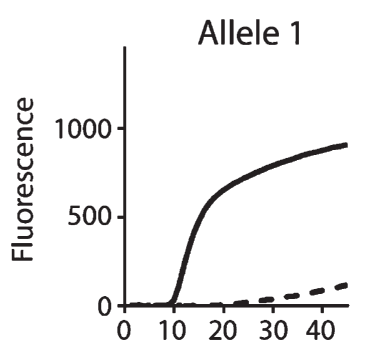

Predominantly 2

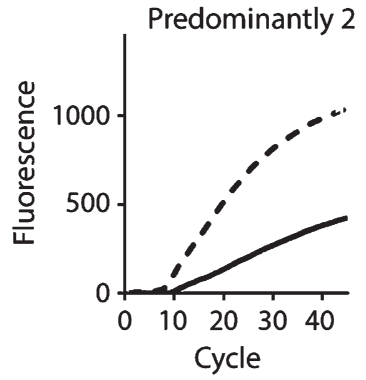

Amplification curves nested KASPar

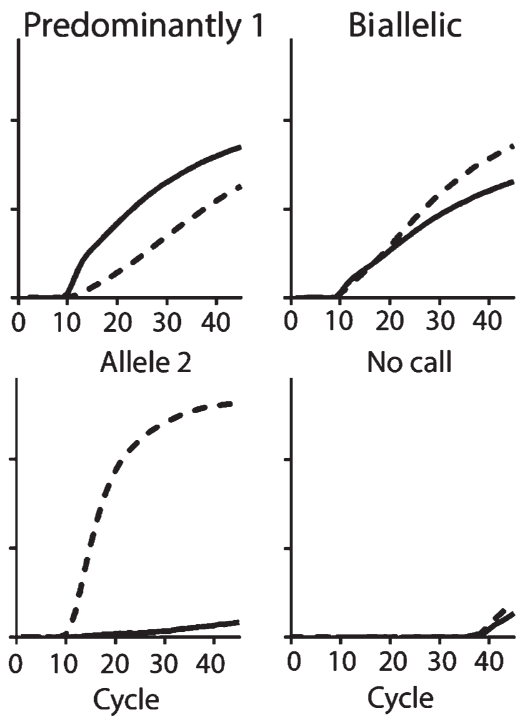

C
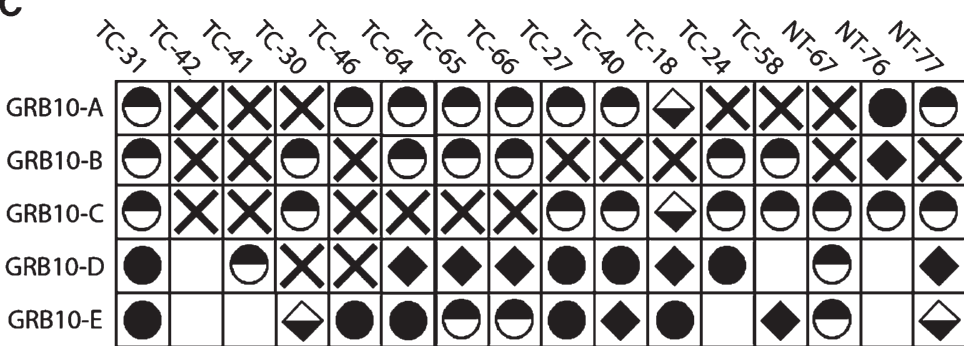

D

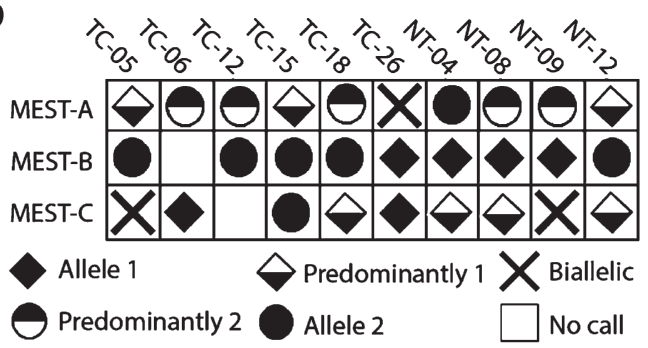

Figure 4. Isoform-dependent ASE detection of GRB10 and MEST. Isoform-specific PCRs were performed, after which KASPar analysis of cSNPs was performed to detect ASE. Cluster analysis of the endpoint fluorescence of the KASPar assay showed five distinct clusters (A). These clusters corresponded to the five different amplification patterns: monoallelic or predominantly monoallelic expression of both alleles and biallelic expression (B). Graphic representation of nested-KASPar ASE-calls shows either monoallelic or predominantly monoallelic expression for assays GRB10-D and GRB10-E (C). MEST showed monoallelic expression with MEST-B assay for all heterozygous samples (D). $A$ and $B$ are the result of nested-KASPar analysis using the GRB10-A amplicon.

derived from a follicular thyroid carcinoma [32]. This cell line has four copies of chromosome 7 with balanced maternal and paternal copies (supplementary material, Figure S6).

To confirm that the siRNA-mediated knockdown was on-target, we performed deconvolution of the SMARTpools and qPCR validation of knockdown on both SMARTpool and single-siRNA transfected cells (Figure 5A, B). Both individual siRNAs and SMARTpools showed a clear reduction of gene expression after siRNA-mediated knockdown (Figure 5B). The single siRNAs also showed similar effects on cell proliferation to the SMARTpools (Figure 5A). Of note, for GRB10, all single siRNAs showed a smaller effect on mRNA expression than the SMARTpools. However, since all siRNAs show a decrease in gene expression of GRB10 and all single siRNAs also show an effect similar to the SMARTpool in the proliferation assay, we concluded that the GRB10 knockdown is on-target. For MEST, two of the single siRNAs did not show a strong effect on proliferation, which for one of these was explained by the lack of mRNA knockdown in the qPCR. Gene expression levels in all samples treated with either the SMARTpool or the single siRNAs targeting
$K L F 14$ were below the detection level of our qPCR. The mock-treated sample and KIFII and scrambled siRNA-treated samples all showed KLF14 expression similar to that of untreated TT2609-C02 cells.

Having confirmed that the siRNA knockdown is on-target and results in mRNA knockdown of the target genes, we set out to investigate the cellular effect of knockdown of the target genes. We compared the cell cycle distributions of cells treated with target siRNAs with those of cells treated with scrambled siRNAs (Figure 5C, D). The strongest effect on cell cycle was observed in the MEST-knockdown cells. These cells showed an increase of $\mathrm{G}_{1}$-phase cells of $17 \%$, together with a decrease of S-phase cells of $15 \%$. This suggests that these cells move slower through $\mathrm{G}_{1}$ or have difficulty with $\mathrm{G}_{1}$ to $\mathrm{S}$-phase transition. Interestingly, knockdown of GRB10, KLF14, PEG10, and CALCR also resulted in an increase in $\mathrm{G}_{1}$-phase cells, albeit to a lesser extent than MEST knockdown. These samples also all showed a decrease in S-phase cells. This indicates that these five genes all result in a decreased growth speed, which fits with the observation that homozygosity on chromosome 7 would be selected against in thyroid cancer. 
A

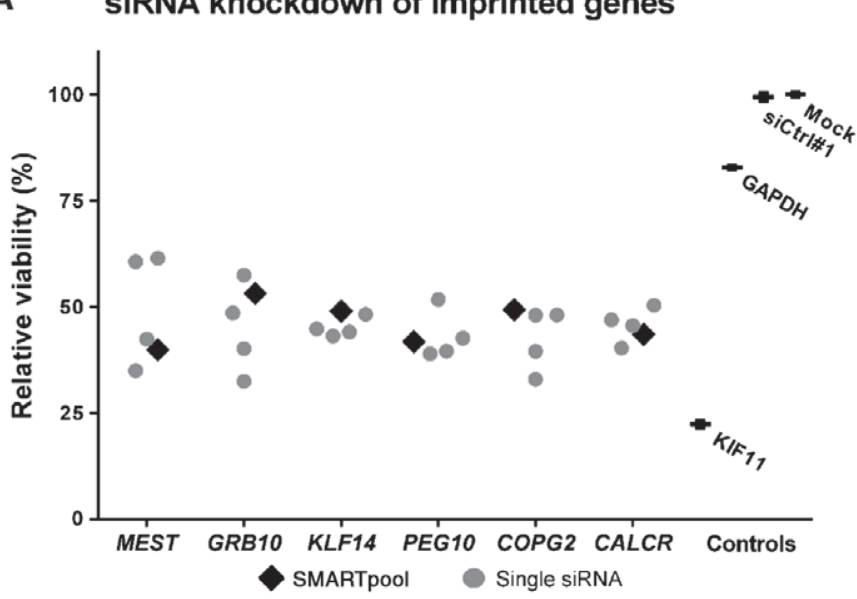

C

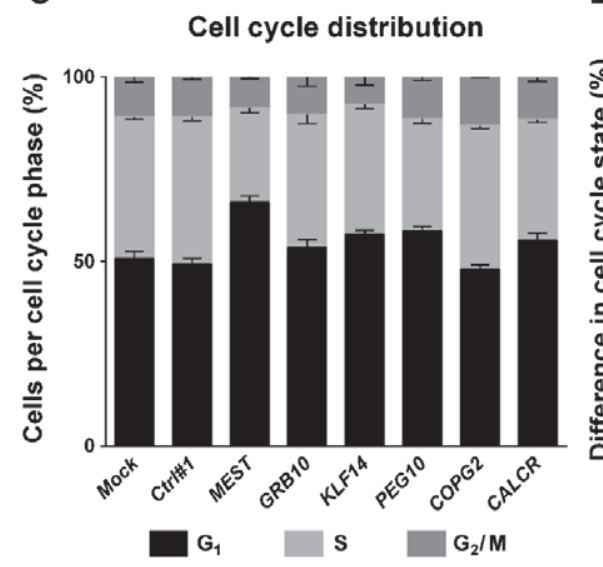
Change in cell cycle distribution

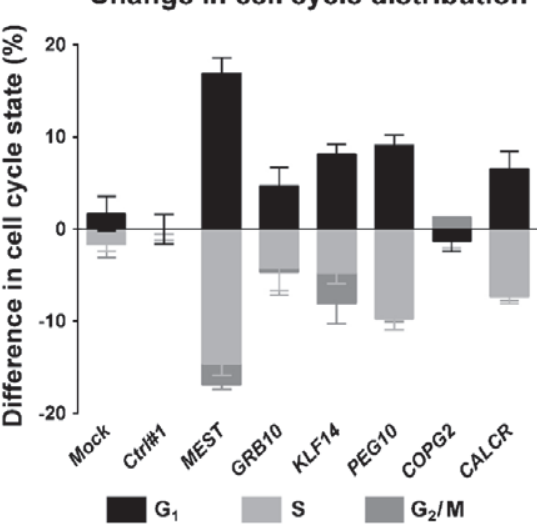

mRNA levels after siRNA knockdown

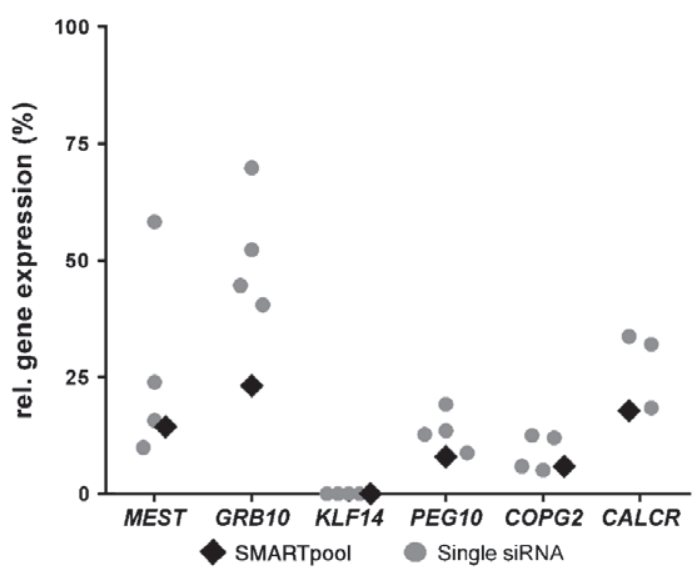

E

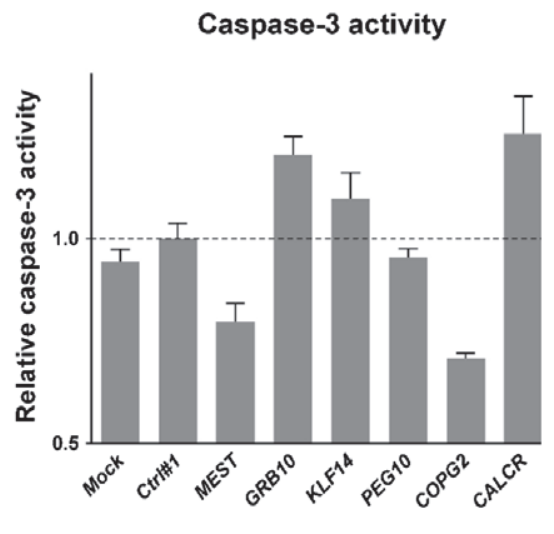

Figure 5. siRNA-mediated knockdown. (A) siRNA knockdown effect on proliferation. (B) RNA expression after siRNA knockdown using SMARTpools and single siRNAs. (C) Percentage of cells in each cell cycle state after siRNA knockdown. (D) Change in cell cycle distribution relative to the scrambled control siRNA (Ctrl\#1) treated cells. (E) Caspase-3 activity was measured to quantify cells undergoing apoptosis after siRNA knockdown. Bars represent the mean with the standard error of the mean.

To investigate whether knockdown of the imprinted cell survival genes also results in cell death, we performed a kinetic assay for caspase-3 activity. In the GRB10-, CALCR-, and KLF14-knockdown samples, we observed up-regulation of active caspase-3, indicative of an increase in apoptotic cells (Figure 5E). Conversely, a reduction in the base-level apoptotic activity was observed in MEST- and COPG2-knockdown cells. MEST knockdown has already been shown to result in a strong $\mathrm{G}_{1}$-phase cell cycle arrest, maybe preventing cells from entering into apoptosis. Knockdown of COPG2, on the other hand, did not show a very strong cell cycle arrest.

\section{Discussion}

Most oncocytic follicular thyroid carcinomas show near-homozygous genomes, but homozygosity of chromosome 7 is never observed [10-12]. These observations led to the hypothesis that there is a set of monoallelically expressed genes on both copies of chromosome 7 that obligate the retention of heterozygosity in this chromosome. We have demonstrated that knockdown of CALCR, COPG2, GRB10, KLF14, MEST, and $P E G 10$ results in reduced cell proliferation, as well as cell cycle arrest and apoptosis. We therefore propose that these six genes are important cell survival genes driving the retention of heterozygosity on chromosome 7 in oncocytic follicular thyroid carcinomas (Figure 6).

A comparable mechanism has been described in paraganglioma. The whole-chromosomal loss of the maternal copy of chromosome 11 in paraganglioma was shown to result in functional loss of the SDHD gene in samples with a paternally transmitted mutation of the gene. Maternal transmission of the SDHD mutation does not result in paraganglioma formation. As SDHD is not imprinted in these cells, the parent-of-origin effect observed here is not explained by SDHD itself. Therefore, it was concluded that the homozygosity of chromosome 11 was essential for paraganglioma formation, not only for the second hit on SDHD, but also for loss of the maternally expressed genes of the chr11p15.5 imprinting cluster [33].

The allele-specific dependency of gene expression is quintessential to our hypothesis. Therefore, we chose to 

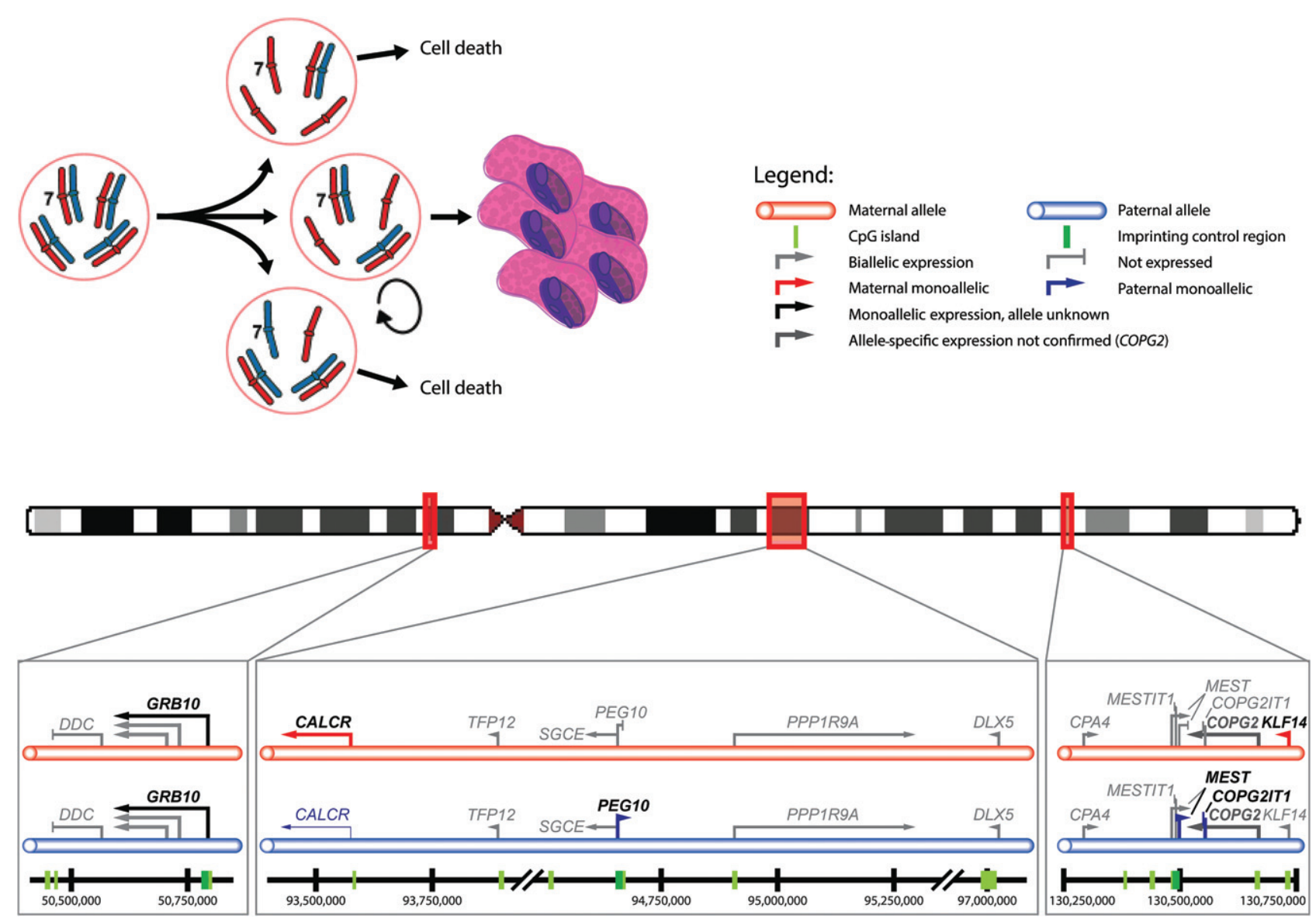

Figure 6. Proposed model for monoallelically-expressed cell survival genes that enforce the retention of chromosome 7.

identify genes regulated by monoallelic DNA methylation, as DNA methylation is stably transmitted to future generations of cells. However, during tumourigenesis, DNA methylation changes are a common occurrence. Also, imprinted genes may lose their imprinting status, a process referred to as loss of imprinting. For example, the IGF2 gene undergoes loss of imprinting in multiple tumour types [34-36]. DNA methylation analysis directed us to the three imprinting clusters on chromosome 7 and confirmed that they are not subjected to loss of imprinting in oncocytic follicular thyroid carcinomas. These imprinting clusters contain genes known to be monoallelically expressed from the paternal and maternal alleles [37-41].

We identified six imprinted cell survival genes CALCR, COPG2, GRB10, KLF14, MEST, and $P E G 10$ - displaying allele-specific expression in thyroid cancer cells. These genes were shown to be essential for tumour cell survival. Transcription of all of these genes was increased in the tumour tissues compared with the normal samples. Up-regulation of MEST and GRB10 was previously described in a pan-cancer analysis, emphasizing their importance in a wide range of tumour types [42]. GRB10 encodes a growth-receptor bound protein that has functions in multiple key signalling pathways, such as Wnt and Akt signalling [40,43]. As an Akt co-activator, GRB10 acts as a potential oncogene, whereas its role as an inhibitor of Wnt and IGF signalling suggests a tumour-suppressor function. This duality might be due to the distinct functions of the different GRB10 isoforms.

MEST also encodes several isoforms, which are isoform-specifically imprinted $[44,45]$. Our results have confirmed monoallelic gene expression of the two isoforms that are transcribed upstream of MESTIT1. MEST has been ascribed to function as a negative regulator of differentiation and an enhancer of cell growth [46,47]. A role for MEST in CRC and lung cancer susceptibility and progression has been proposed previously [48]. MEST has also been implicated in the regulation of ASE of $C O P G 2$, which is located downstream. MacIsaac et al have shown that MESTXL, a $>10 \mathrm{~kb}$ isoform of $M E S T$, which runs into the COPG2 locus, can influence allelic usage of COPG2 [49].

$K L F 14$ was recently investigated in the context of tumourigenesis. KLF14 expression is reduced in diverse cancer types, and a KLF14-knockdown mouse strain was shown to be prone to the development of tumours, but no thyroid carcinomas were reported. Contrastingly, a recent study showed that KLF14 down-regulation can induce mitotic catastrophe and apoptosis [50]. Together with our results showing induction of apoptosis after KLF14 knockdown, this suggests that KLF14 has oncogenic activity in thyroid cancers. 
Loss of most of the imprinted ASE target genes resulted in an increase in the fraction of cells in the $\mathrm{G}_{1}$-phase of the cell cycle. In support of our observations, recent in vitro experiments identified PEG10 to be pivotal in the cell cycle progression of neuroendocrine prostate cancer [51], and loss of PEG10 was shown to reduce proliferation and invasion in trophoblasts [52]. PEG10 expression is increased in liver and gallbladder cancers [53,54], as well as in acute and chronic B-cell leukaemia [55].

In addition to the genes that we examined in our experiments, the imprinting clusters on chromosome 7 also contain non-protein-coding genes. We tested the expression levels of the two internal transcripts MESTIT1 and COPG2IT1. MESTIT1 was exclusively expressed in medullary thyroid cancer (supplementary material, Figure S4). COPG2IT1, on the other hand, was expressed in all thyroid cancer samples. The only reason why it was not investigated further was the lack of availability of siRNAs targeting non-coding genes. Additionally, a recent publication showed that miR-335 is also co-regulated with MEST. This suggests that miR-335 is possibly up-regulated in thyroid cancer, as our results show that MEST is up-regulated.

Further research is required to investigate whether there are additional monoallelically expressed cell survival genes on chromosome 7 . These studies should include miRNAs such as miR-335, transcribed from intron 2 of MEST, and shown to be imprinted and expressed from the same allele as MEST [47]. Two recent studies have examined the expression levels of miRNAs in thyroid tumours; miR-335 was shown to be up-regulated in follicular carcinomas compared with follicular adenomas [56].

Several other tumour types also show preferential retention of heterozygosity on chromosome 7 . Therefore, cell survival genes are possibly equally important in a large range of tumour types, as well as for the normal cell types that these tumours originate from. Therefore, to explore the therapeutic potential of these genes, their expression should be explored. We also note, though, that if these genes are expressed in normal tissues, this does not exclude the possibility of cancer cells having an acquired dependency on these genes that is not present in the corresponding normal tissues.

This work further supports the essential role of genes located in chromosome 7 for cell survival. We provide a rationale for the infrequent targeting of this chromosome by gross mechanisms of chromosomal instability.

\section{Acknowledgement}

This work was supported by the EUROTRANS-BIO project FAST-SEQ.

\section{Author contribution statement}

The authors contributed in the following way: AB, HM, and TvW: conceived and designed the experiments; $\mathrm{AB}$ and NFdM: performed the experiments; JO, YZ,
WC, and BvdW: contributed materials/analysis tools; $\mathrm{AB}$ and JO: analysed the data: $\mathrm{AB}$ and TvW: drafted the manuscript. All authors read and approved the final manuscript.

\section{References}

Note: References 57-63 are cited in the supporting information to this article.

1. Murnane JP. Telomere dysfunction and chromosome instability. Mutat Res 2012; 730: 28-36.

2. Bakhoum SF, Kabeche L, Murnane JP, et al. DNA-damage response during mitosis induces whole-chromosome missegregation. Cancer Discov 2014; 4: 1281-1289.

3. Lee S, Kopp F, Chang TC, et al. Noncoding RNA NORAD regulates genomic stability by sequestering PUMILIO proteins. Cell 2016; 164: 69-80.

4. Burrell RA, McClelland SE, Endesfelder D, et al. Replication stress links structural and numerical cancer chromosomal instability. Nature 2013; 494: 492-496.

5. The Cancer Genome Atlas Network. Integrated genomic characterization of papillary thyroid carcinoma. Cell 2014; 159: 676-690.

6. Pisanu A, Di Chiara B, Reccia I, et al. Oncocytic cell tumors of the thyroid: factors predicting malignancy and influencing prognosis, treatment decisions, and outcomes. World J Surg 2010; 34: 836-843.

7. Dettmer M, Schmitt A, Steinert H, et al. Poorly differentiated oncocytic thyroid carcinoma - diagnostic implications and outcome. Histopathology 2012; 60: 1045-1051.

8. Cannon J. The significance of Hürthle cells in thyroid disease. Oncologist 2011; 16: 1380-1387.

9. Corver WE, Ruano D, Weijers K, et al. Genome haploidisation with chromosome 7 retention in oncocytic follicular thyroid carcinoma. PLoS One 2012; 7: e38287.

10. Corver WE, van Wezel T, Molenaar K, et al. Near-haploidization significantly associates with oncocytic adrenocortical, thyroid, and parathyroid tumors but not with mitochondrial DNA mutations. Genes Chromosomes Cancer 2014; 53: 833-844.

11. Wagle N, Grabiner BC, Van Allen EM, et al. Response and acquired resistance to everolimus in anaplastic thyroid cancer. $N$ Engl J Med 2014; 371: 1426-1433.

12. Kasaian K, Chindris AM, Wiseman SM, et al. MEN1 mutations in Hürthle cell (oncocytic) thyroid carcinoma. J Clin Endocrinol Metab 2015; 100: E611-E615.

13. Holmfeldt L, Wei L, Diaz-Flores E, et al. The genomic landscape of hypodiploid acute lymphoblastic leukemia. Nature Genet 2013; 45: $242-252$.

14. Lo KC, Bailey D, Burkhardt T, et al. Comprehensive analysis of loss of heterozygosity events in glioblastoma using the $100 \mathrm{~K}$ SNP mapping arrays and comparison with copy number abnormalities defined by BAC array comparative genomic hybridization. Genes Chromosomes Cancer 2008; 47: 221-237.

15. Bovee JV, van Royen M, Bardoel AF, et al. Near-haploidy and subsequent polyploidization characterize the progression of peripheral chondrosarcoma. Am J Pathol 2000; 157: 1587-1595.

16. Schindler G, Capper D, Korshunov A, et al. Spinal metastasis of gliosarcoma: array-based comparative genomic hybridization for confirmation of metastatic spread. J Clin Neurosci 2014; 21: $1945-1950$.

17. Yuan ZR, Wang R, Solomon J, et al. Identification and characterization of survival-related gene, a novel cell survival gene controlling apoptosis and tumorigenesis. Cancer Res 2005; 65: 10716-10724.

18. Nambiar S, Mirmohammadsadegh A, Hassan M, et al. Identification and functional characterization of $A S K / D b f 4$, a novel cell survival 
gene in cutaneous melanoma with prognostic relevance. Carcinogenesis 2007; 28: 2501-2510.

19. Thompson JM, Nguyen QH, Singh M, et al. Approaches to identifying synthetic lethal interactions in cancer. Yale J Biol Med 2015; 88: $145-155$.

20. Hoftijzer H, Heemstra KA, Morreau H, et al. Beneficial effects of sorafenib on tumor progression, but not on radioiodine uptake, in patients with differentiated thyroid carcinoma. Eur $J$ Endocrinol 2009; 161: 923-931.

21. Du P, Kibbe WA, Lin SM. lumi: a pipeline for processing Illumina microarray. Bioinformatics 2008; 24: 1547-1548.

22. Touleimat N, Tost J. Complete pipeline for Infinium ${ }^{\circledR}$ Human Methylation $450 \mathrm{~K}$ BeadChip data processing using subset quantile normalization for accurate DNA methylation estimation. Epigenomics 2012; 4: $325-341$

23. van Roon EH, Boot A, Dihal AA, et al. BRAF mutation-specific promoter methylation of FOX genes in colorectal cancer. Clin Epigenetics 2013; 5: 2.

24. Pfaffl MW. A new mathematical model for relative quantification in real-time RT-PCR. Nucleic Acids Res 2001; 9: 29.

25. Vichai V, Kirtikara K. Sulforhodamine B colorimetric assay for cytotoxicity screening. Nature Protoc 2006; 1: 1112-1116.

26. van Haaften C, Boot A, Corver WE, et al. Synergistic effects of the sesquiterpene lactone, EPD, with cisplatin and paclitaxel in ovarian cancer cells. J Exp Clin Cancer Res 2015; 34: 38.

27. Morison IM, Reeve AE. A catalogue of imprinted genes and parent-of-origin effects in humans and animals. Hum Mol Genet 1998; 7: 1599-1609.

28. Jirtle RL. Genomic imprinting and cancer. Exp Cell Res 1999; 248: $18-24$.

29. Huntriss JD, Hemmings KE, Hinkins M, et al. Variable imprinting of the MEST gene in human preimplantation embryos. Eur J Hum Genet 2013; 21: 40-47.

30. Arnaud P, Monk D, Hitchins M, et al. Conserved methylation imprints in the human and mouse GRB10 genes with divergent allelic expression suggests differential reading of the same mark. Hum Mol Genet 2003; 12: 1005-1019.

31. Monk D, Arnaud P, Frost J, et al. Reciprocal imprinting of human $G R B 10$ in placental trophoblast and brain: evolutionary conservation of reversed allelic expression. Hum Mol Genet 2009; 18: 3066-3074.

32. Geldof AA, Versteegh LRT, van Mourik JC, et al. Clonally related but phenotypically divergent human cancer cell lines derived from a single follicular thyroid cancer recurrence (TT2609). Thyroid 2001; 11: $909-917$

33. Hensen EF, Jordanova ES, van Minderhout IJ, et al. Somatic loss of maternal chromosome 11 causes parent-of-origin-dependent inheritance in SDHD-linked paraganglioma and phaeochromocytoma families. Oncogene 2004; 23: 4076-4083.

34. Tian F, Tang Z, Song G, et al. Loss of imprinting of IGF2 correlates with hypomethylation of the $\mathrm{H} 19$ differentially methylated region in the tumor tissue of colorectal cancer patients. Mol Med Rep 2012; 5: $1536-1540$.

35. Murata A, Baba Y, Watanabe M, et al. IGF2 DMR0 methylation, loss of imprinting, and patient prognosis in esophageal squamous cell carcinoma. Ann Surg Oncol 2014; 21: 1166-1174.

36. Ozaki S, Kawahara E, Maenaka S, et al. Distinct allelic expression patterns of imprinted IGF2 in adenocarcinoma and squamous cell carcinoma of the lung. Oncol Lett 2014; 8: 2561-2564.

37. Baran Y, Subramaniam M, Biton A, et al. The landscape of genomic imprinting across diverse adult human tissues. Genome Res 2015; 7: 927-936.

38. Yamada T, Mitsuya K, Kayashima T, et al. Imprinting analysis of 10 genes and/or transcripts in a 1.5-Mb MEST-flanking region at human chromosome 7q32. Genomics 2004; 83: 402-412.
39. Blagitko N, Mergenthaler S, Schulz U, et al. Human GRB10 is imprinted and expressed from the paternal and maternal allele in a highly tissue- and isoform-specific fashion. Hum Mol Genet 2000; 9: $1587-1595$.

40. Uribe-Lewis S, Woodfine K, Stojic L, et al. Molecular mechanisms of genomic imprinting and clinical implications for cancer. Expert Rev Mol Med 2011; 13: e2.

41. Hannula-Jouppi K, Muurinen M, Lipsanen-Nyman M, et al. Differentially methylated regions in maternal and paternal uniparental disomy for chromosome 7. Epigenetics 2014; 9: 351-365.

42. Kim J, Bretz CL, Lee S. Epigenetic instability of imprinted genes in human cancers. Nucleic Acids Res 2015; 22: 10689-10699.

43. Jahn T, Seipel P, Urschel S, et al. Role for the adaptor protein Grb10 in the activation of Akt. Mol Cell Biol 2002; 22: 979-991.

44. Kosaki K, Kosaki R, Craigen WJ, et al. Isoform-specific imprinting of the human PEG1/MEST gene. Am J Hum Genet 2000; 66: 309-312.

45. Stevens AJ, Stuffrein-Roberts S, Cree SL, et al. G-quadruplex structures and $\mathrm{CpG}$ methylation cause drop-out of the maternal allele in polymerase chain reaction amplification of the imprinted MEST gene promoter. PLoS One 2014; 9: e113955.

46. Karbiener M, Glantschnig C, Pisani DF, et al. Mesoderm-specific transcript (MEST) is a negative regulator of human adipocyte differentiation. Int J Obes (Lond) 2015; 12: 1733-1741.

47. Hiramuki Y, Sato T, Furuta Y, et al. Mest but not miR-335 affects skeletal muscle growth and regeneration. PLoS One 2015; 10: e0130436.

48. Kohda M, Hoshiya H, Katoh M, et al. Frequent loss of imprinting of IGF2 and MEST in lung adenocarcinoma. Mol Carcinog 2001; 31: 184-191.

49. MacIsaac JL, Bogutz AB, Morrissy AS, et al. Tissue-specific alternative polyadenylation at the imprinted gene Mest regulates allelic usage at Copg2. Nucleic Acids Res 2012; 40: 1523-1535.

50. Fan G, Sun L, Shan P, et al. Loss of KLF14 triggers centrosome amplification and tumorigenesis. Nature Commun 2015; 6: 8450

51. Akamatsu S, Wyatt AW, Lin $\mathrm{D}$, et al. The placental gene PEG10 promotes progression of neuroendocrine prostate cancer. Cell Rep 2015; 12: 922-936.

52. Chen H, Sun M, Liu J, et al. Silencing of paternally expressed gene 10 inhibits trophoblast proliferation and invasion. PLoS One 2015; 10: $\mathrm{e} 0144845$.

53. Wang C, Xiao Y, Hu Z, et al. PEG10 directly regulated by E2Fs might have a role in the development of hepatocellular carcinoma. FEBS Lett 2008; 582: 2793-2798.

54. Liu DC, Yang ZL, Jiang S. Identification of PEG10 and TSG101 as carcinogenesis, progression, and poor-prognosis related biomarkers for gallbladder adenocarcinoma. Pathol Oncol Res 2011; 17: $859-866$.

55. Hu C, Xiong J, Zhang L, et al. PEG10 activation by co-stimulation of CXCR5 and CCR7 essentially contributes to resistance to apoptosis in $\mathrm{CD} 19^{+} \mathrm{CD} 34^{+} \mathrm{B}$ cells from patients with B cell lineage acute and chronic lymphocytic leukemia. Cell Mol Immunol 2004; 1: 280-294.

56. Rossing M, Borup R, Henao R, et al. Down-regulation of microRNAs controlling tumourigenic factors in follicular thyroid carcinoma. $J$ Mol Endocrinol 2012; 48: 11-23.

57. Goeman JJ, van de Geer SA, de Kort F, et al. A global test for groups of genes: testing association with a clinical outcome. Bioinformatics 2004; 20: 93-99.

58. Hannula-Jouppi K, Muurinen M, Lipsanen-Nyman M, et al. Differentially methylated regions in maternal and paternal uniparental disomy for chromosome 7. Epigenetics 2014; 9: 351-365.

59. Daca-Roszak P, Pfeifer A, Zebracka-Gala J, et al. Impact of SNPs on methylation readouts by Illumina Infinium HumanMethylation450 BeadChip Array: implications for comparative population studies. BMC Genomics 2015; 16: 1003. 
60. Lapa C, Werner RA, Schmid JS, et al. Prognostic value of positron emission tomography-assessed tumor heterogeneity in patients with thyroid cancer undergoing treatment with radiopeptide therapy. $\mathrm{Nucl}$ Med Biol 2015; 42: 349-354.

61. Corver WE, Ruano D, Weijers K, et al. Genome haploidisation with chromosome 7 retention in oncocytic follicular thyroid carcinoma. PLoS One 2012; 7: e38287.
62. Corver WE, van Wezel T, Molenaar K, et al. Near-haploidization significantly associates with oncocytic adrenocortical, thyroid, and parathyroid tumors but not with mitochondrial DNA mutations. Genes Chromosomes Cancer 2014; 53: 833-844.

63. Humphray SJ, Oliver K, Hunt AR, et al. DNA sequence and analysis of human chromosome 9. Nature 2004; 429: 369-374.

\section{SUPPLEMENTARY MATERIAL ONLINE \\ Supplementary materials and methods \\ Supplementary figure legends}

Figure S1. Distribution of probes over the genome before and after applying the $50 \%$ intensity filter.

Figure S2. BMIQ-normalization output for each of the samples.

Figure S3. $\beta$-values of loci with hemi-methylation on chromosome 7 in thyroid cancer.

Figure S4. Gene expression levels for the 15 imprinted genes annotated to the imprinting clusters on chromosome 7.

Figure S5. Schematic display of the GRB10 (A) and MEST (B) loci, including the isoform-specific amplification amplicons used for the nested KASPar analysis of allele-specific expression and the cSNPs analysed.

Figure S6. LAIR analysis of TT2609-C02.

Figure S7. Percentage of hemi-methylated probes for the non-NHG (A) and NHG (B) groups per megabase.

Figure S8. Distribution of consistent hemi-methylation on chromosome 7 in all thyroid cancer samples.

Figure S9. Distribution of hemi-methylation over the genome.

Table S1. Overview of samples used in this study.

Table S2. Complete list of the primers used in this study, including KASPar and M13-tail sequences where applicable.

Table S3. Details of the Dharmacon siRNAs used in this study, including siRNA sequences.

Table S4. Detailed allele-specific expression (ASE) results.

\section{Years ago in the Journal of Pathology...}

The effect of 9,10-dimethyl-1,2-benzanthracene on the skin and cysts of "cyst-bearing" Merino sheep

L. C. Lloyd

To view these articles, and more, please visit:

www.thejournalofpathology.com

Click 'ALL ISSUES (1892 - 2016)', to read articles going right back to Volume 1 , Issue 1.

\section{The Journal of Pathology Understanding Disease}

\section{A Journal of}

The Pathological Society 